\section{Estrategia educativa sobre las consecuencias de relación sexual precoz en adolescentes de la comunidad "El Carmen"}

\author{
Educational strategy on the consequences of early sexual \\ relations in adolescents from the "El Carmen" community

\section{Estratégia de ensino sobre as consequências das relações sexuais precoces em adolescentes da comunidade "O Carmen"}

\author{
Sharon Matos \\ drasharonm@gmail.com
}

Recibido diciembre 2019 / Revisión enero 2020 / Aceptado 1 de mayo 2020

\section{RESUMEN}

Introducción: La adolescencia es el periodo en el que los jóvenes comienzan a sentir curiosidad por la sexualidad y su naturaleza. La educación sexual en los adolescentes es un asunto urgente en América, y en especial en Venezuela, Objetivo: diseñar un programa de intervención educativa sobre las consecuencias de las relaciones sexuales precoces en el periodo comprendido 2017-2018. Método: estudio prospectivo, cuasiexperimental con pre test y post test de intervención educativa. La población estuvo constituida por 100 adolescentes registrados en la comunidad el Carmen y la muestra 30 adolescentes. El instrumento fue un cuestionario sobre conocimiento sobre las consecuencias de la relación sexual precoz. Se utilizó estadística descriptiva, los datos se expresaron en tablas. Resultados: Se encontró un $66,6 \%$ de los adolescentes con déficit de conocimientos, posterior a la intervención educativa se obtuvo un $89,99 \%$ que respondieron de forma acertada. Conclusión: el aumento del porcentaje de respuestas correctas hace inferir que la intervención educativa fue favorable y hubo en los adolescentes aumento el dominio cognitivo sobre las consecuencias de la relación sexual precoz. Es necesario reevaluar las estrategias a fin de mejorar el porcentaje de las respuestas en los estudiantes.

Palabras clave: Adolescencia; salud sexual; anticonceptivo, educación

\begin{abstract}
Introduction: Adolescence is the period in which young people begin to be curious about sexuality and its nature. Sex education in adolescents is an urgent matter in America, and especially in Venezuela, Objective: to design an educational intervention program on the consequences of early sexual relations in the period from 2017 to 2018. Method: prospective, quasi-experimental study with pre-test and post-test of educational intervention. The population consisted of 100 adolescents registered in the El Carmen community and the sample 30 adolescents. The instrument was a questionnaire about knowledge about the consequences of early sexual intercourse. Descriptive statistics were used, the data were expressed in tables. Results: $66.6 \%$ of the adolescents with knowledge deficits were found. After the educational intervention, $89.99 \%$ were found to have responded correctly. Conclusion: the increase in the percentage of correct responses leads us to infer that the educational intervention was favorable and that there was an increase in cognitive dominance of the consequences of early sexual intercourse in adolescents. Strategies need to be reevaluated to improve the percentage of student responses.
\end{abstract}

Key words: Adolescence; sexual health; contraception, education
SM: Universidad de la Ciencia de la Salud, Venezuela.

Postgrado en Medicina General Integral en la misma universidad. Actualmente se encuentra laborando en el CDI "Cesar Augusto Ríos" como médico especialista tipo I. 
SM: Universidad de la Ciencia de la Salud, Venezuela. Postgrado en Medicina General Integral en la misma universidad. Actualmente se encuentra laborando en el CDI "Cesar Augusto Ríos" como médico especialista tipo I.

\section{RESUMO}

Introdução: A adolescência é o período em que os jovens começam a ter curiosidade sobre a sexualidade e sua natureza. A educação sexual em adolescentes é uma questão urgente na América, e especialmente na Venezuela. Objetivo: elaborar um programa de intervenção educacional sob as consequências das relações sexuais precoces no período de 2017 à 2018. Materiais e Método: estudo prospectivo, quase-experimental, com pré-teste e pós-teste de intervenção educacional. A população foi composta por 100 adolescentes cadastrados na comunidade de "O Carmen" e a amostra foi de 30 adolescentes. $O$ instrumento foi um questionário sobre o conhecimento das consequências da relação sexual precoce. Utilizou-se estatística descritiva, os dados foram sistematizados em tabelas. Resultados: o $66,6 \%$ dos adolescentes apresentaram déficit de conhecimento e, após a intervenção educativa, $89,99 \%$ responderam corretamente as questões. Conclusão: o aumento no percentual de respostas corretas nos leva a inferir que a intervenção educacional foi favorável e houve um aumento no domínio cognitivo sobre as consequências da relação sexual precoce em adolescentes. É necessário reavaliar as estratégias para melhorar o percentual de respostas nos alunos.

Palabras clave: Adolescência; saúde sexual; contraceptivo; educação

\section{INTRODUCCIÓN}

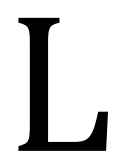
a sexualidad es un componente del ser humano y parte esencial de la salud integral, se desarrolla mediante la interacción entre la persona y las estructuras sociales. El desarrollo pleno de esta dimensión humana es esencial para el bienestar individual, colectivo e interpersonal.

En cada etapa del desarrollo humano, la sexualidad tiene manifestaciones diferentes como en la adolescencia que representa una delicada transición en la que los jóvenes desarrollan su personalidad y construyen su identidad. Es precisamente en este periodo en el que los jóvenes comienzan a sentir curiosidad por la sexualidad y su naturaleza. El lugar que los adolescentes tienen asignados en la sociedad y la forma cómo son tratados es determinante para su salud reproductiva; el bienestar físico y psicosocial además contribuye al desarrollo de conductas sexuales y reproductivas saludables.
La adolescencia es definida como una etapa entre la niñez y edad adulta que transcurre entre los 10 y 19 años que cronológicamente se inicia por los cambios puberales y que se caracteriza por profundas transformaciones biológicas psicológicas y sociales muchas de ellas generadoras de crisis, conflictos $y$ contradicciones (1). Se divide en tres etapas, con características diferentes, con formas distintas de enfrentar la sexualidad y un embarazo: adolescencia temprana (10-14 años), adolescencia media (15-16 años) y adolescencia tardía (17-19 años) (10-16) (2).

La adolescencia es una etapa compleja, donde las decisiones y conductas que tomen los y las adolescentes en su salud sexual y reproductiva, dependerán de las oportunidades económicas, laborales, educativas, oferta de anticoncepción, situación socioeconómica, características personales, normas sociales e influencia del medio o contexto social en que viven y crecen (3). El adolescente por lo general no se encuentra maduro en lo psíquico, social y emocional para enfrentar el proceso de 
reproducción, lo que provoca que muchos inicien una actividad sexual precoz, basada fundamentalmente en relaciones sexuales

Es por ello que, el problema del embarazo en la adolescencia no tiene una causa específica, sino que es multideterminado. Varios autores destacan en este grupo, factores de riesgo biológicos y psicosociales. Lo primero se refiere a que las adolescentes con menarquía precoz están más expuestas al riesgo de embarazo, hay un aumento de las relaciones prematrimoniales y el empleo de los métodos anticonceptivos sigue siendo bajo. El segundo aspecto plantea que un mal funcionamiento familiar puede predisponer a una relación sexual prematura (3).

En un estudio se encontró que un 54\% de los adolescentes mantenían relaciones sexuales, dos terceras partes las iniciaron antes de los quince años. Ahora bien, que los jóvenes tengan sus primeros encuentros sexuales durante la adolescencia temprana no significa realmente un problema, porque físicamente se encuentran aptos para realizarlos, el problema es que, la mayor parte de los adolescentes no están preparados psicológica y socialmente para aceptar las consecuencias que se derivan de su actividad sexual, sin dejar de lado que la información sexual en los adolescentes es inexistente o incorrecta, pues la obtienen en el grupo de amigos, de los medios de comunicación y especialmente de Internet, es decir de medios que no son los más idóneos para difundir y orientar (4).

La educación sexual en los adolescentes continúa siendo un asunto urgente en América, y en especial en Venezuela, los abortos en situaciones de riesgo, los embarazos precoces y no deseados, las infecciones de transmisión sexual, promiscuidad, prostitución, abuso sexual, violencia, una vida sexual con aberraciones y la falta de acceso a los anticonceptivos, incita a considerar si realmente se les ofrece a los jóvenes la información oportuna, en el momento indicado, con carácter preventivo.

El Observatorio Venezolano de los Derechos Humanos de las Mujeres, en el informe sobre la Salud Sexual en Venezuela, asume que el embarazo en las adolescentes es el segundo problema de salud sexual y reproductiva a nivel nacional. El 50\% de las adolescentes venezolanas se inician sexualmente antes de los 19 años y $10 \%$ antes de los 15 años. El $21 \%$ de los nacimientos vivos registrados en el país ocurren en adolescentes entre 15 y 19 años, donde dos de cada cinco estudiantes dejan la escuela (5). Estas cifras alarmantes confirman la situación existencial de los adolescentes venezolanos, donde las mujeres y hombres jóvenes tienen relaciones sin tomar en cuenta la prevención, lo cual constituye un riesgo inminente, porque, así como pueden quedar embarazadas por falta de cuidados anticonceptivos, asimismo pueden contraer algunas infecciones de transmisión sexual

Ante la situación planteada, la población adolescente de la comunidad El Carmen no escapa de esta problemática en la precocidad de la sexualidad y sus consecuencias. Por tal motivo se decidió realizar esta investigación que trata de crear las condiciones necesarias para preservar la salud física y mental mediante prácticas educativas y el fomento de estilos de vida adecuados

Por ello, el propósito de la presente investigación fue diseñar una estrategia de intervención educativa para incrementar el conocimiento de los adolescentes de 13 a 16 años de la comunidad "El Carmen 1" municipio Iribarren estado Lara sobre las consecuencias de relación sexual precoz en el periodo 2017-2018. 


\section{MATERIALES Y MÉTODOS}

e realizó un estudio cuasi - experimental con pre test y post test de intervención educativa a los adolescentes de la comunidad el Carmen. La población estuvo constituida por 100 adolescentes registrados en la comunidad con 30 adolescentes de la totalidad como muestra, quienes manifestaron su consentimiento al igual que sus padres y representantes. Se informo acerca de los aspectos bioéticos del estudio. Se aplicó un cuestionario para conocer el nivel de conocimiento que poseen sobre las consecuencias de las relaciones sexuales precoz y se elaboró un programa de educación sexual con base en las carencias detectadas, que se impartió mediante discusiones grupales $\mathrm{y}$ un taller, posteriormente se aplicó nuevamente el mismo cuestionario (Post Test) a la misma muestra de adolescentes. Se solicitó el consentimiento de los jóvenes y sus representantes. Los datos fueron recogidos y llevados a una base de datos, posteriormente, se llevaron a tablas y gráficos.

\section{RESULTADOS Y DISCUSIÓN}

A continuación se presenta el análisis de los resultados arrojados en el estudio acerca de la estrategia educativa sobre las consecuencias de relación sexual precoz en adolescentes de la comunidad "El Carmen", mediante la descripción gráfica de los datos encontrado durante la aplicación del cuestionario sobre el nivel de conocimientos que posee la población objeto de estudio.

Iniciamos con el gráfico 1, en donde se observó que el sexo predominante fue el femenino con $56.6 \%$ en las edades entre 15-16 con un $30 \%$ mientras que el masculino obtuvo el $43.4 \%$ y en las edades comprendidas entre15-16 años con un 26.6\%. La adolescencia, es un período de múltiples cambios tanto físicos como psicológicos, caracterizada por inestabilidad emocional, se observó que el mayor porcentaje de adolescentes encuestados correspondió al grupo etario entre 15 y 16 años de edad y el sexo predominante en ambos grupos fue el femenino.

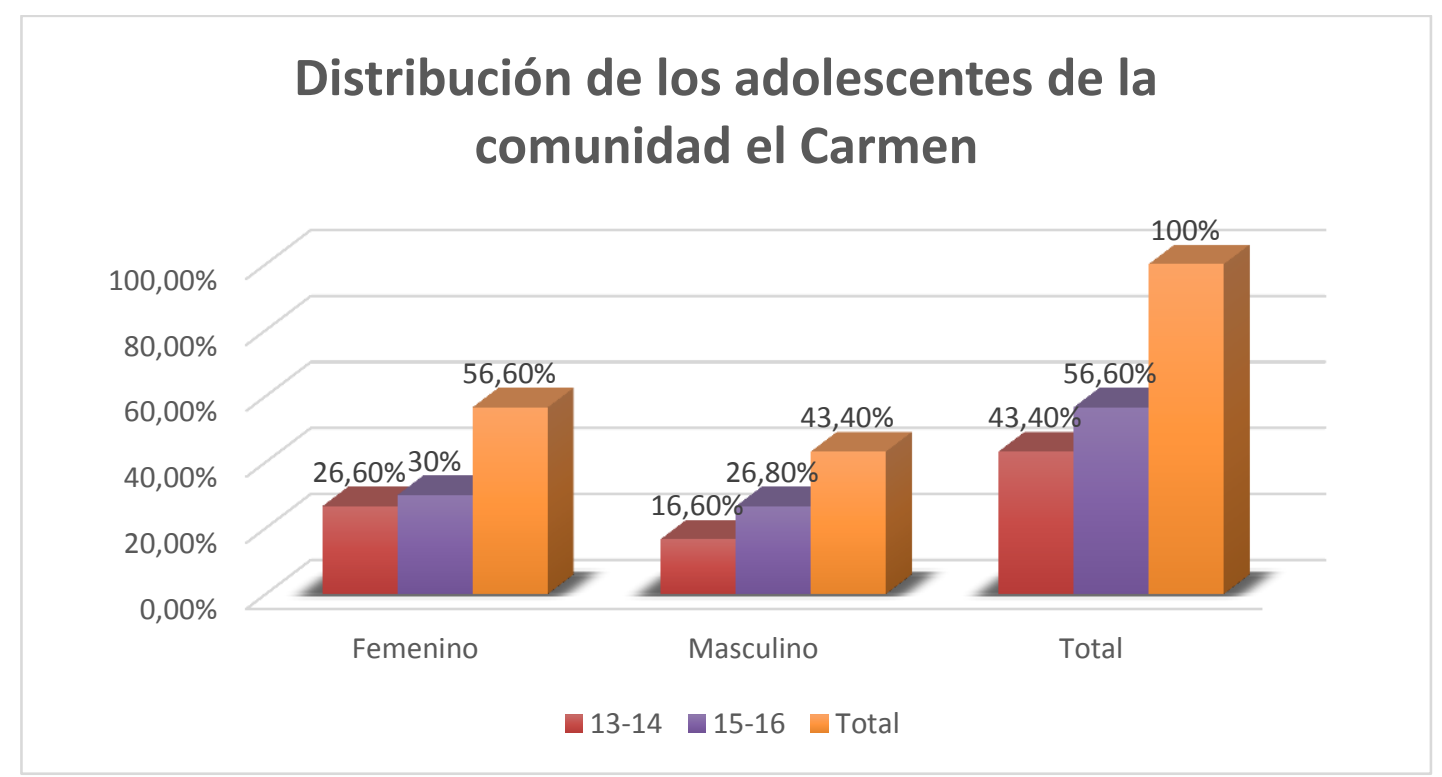

Gráfico 1. Distribución de los adolescentes de la comunidad el Carmen según edad y sexo en el periodo de marzo a septiembre del año 2018. 
Posteriormente, se observó en el gráfico 2 que el $63.33 \%$ de los adolescentes si han iniciado relaciones sexuales, las edades que predominaron fueron los 15 y 16 años con un $33.33 \%$. Mientras que el $36.66 \%$ no han tenido relaciones sexuales. Estos resultados llaman poderosamente la atención debido a que estos adolescentes ya han iniciado sus relaciones sexuales y con ellos los riesgos asociados a esta actividad si no se toman las medidas preventivas.

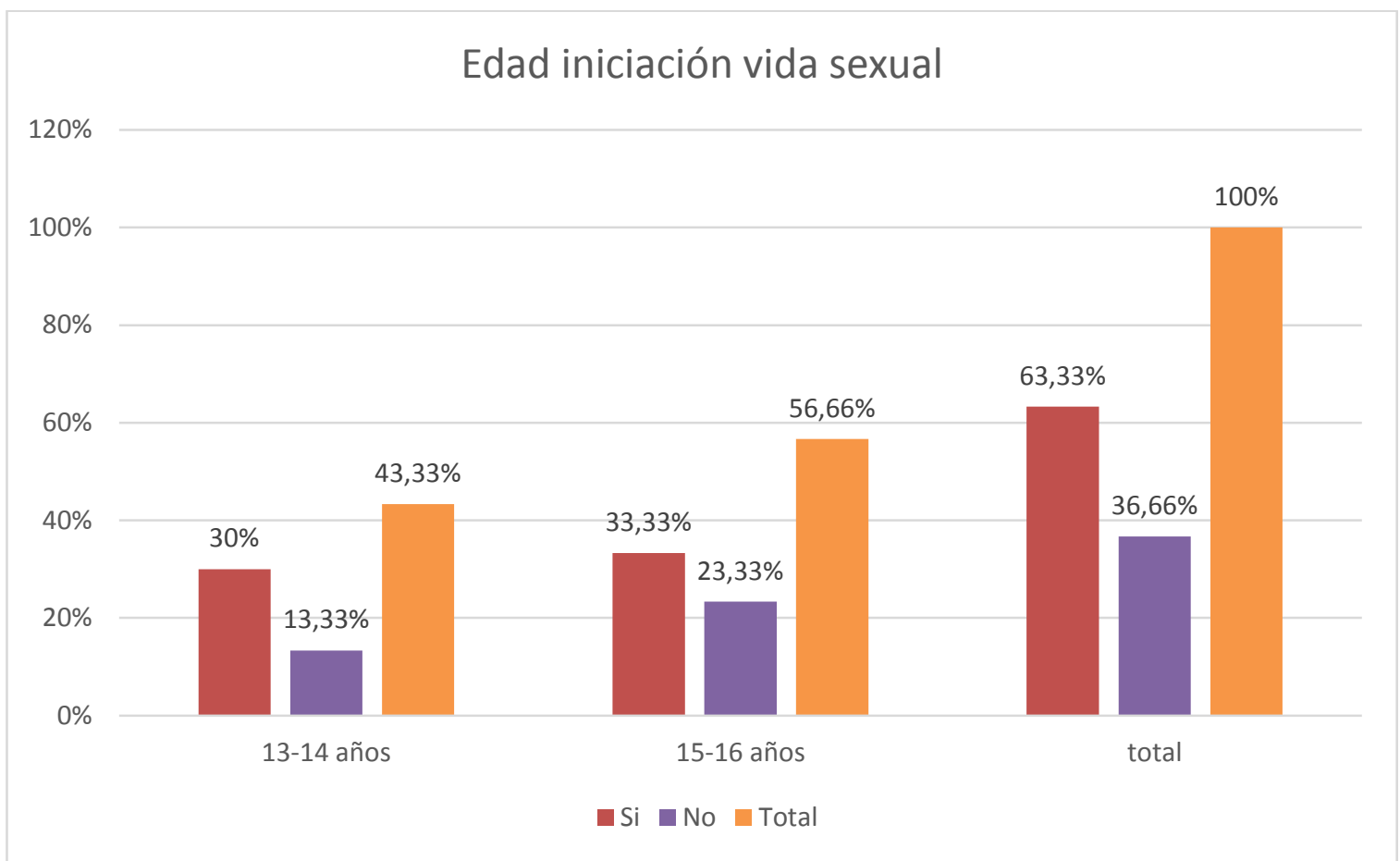

Gráfico 2. Adolescentes entre 13 y 16 años de edad de la comunidad el Carmen, inicio precoz de sexuales. 2018

Entre los factores que promueven el inicio de las relaciones sexuales precoces predominó la falta o mala comunicación con los padres en el sexo femenino con un 43.32\%. En estos resultados se observa que la frecuencia de haber tenido relaciones sexuales era menor en aquellos que frecuentemente habían tratado con los padres cuestiones sobre el amor y el enamoramiento, así como sobre temas de biología sexual, como los cambios sexuales que experimentan. Seguido de la influencia de los medios de comunicación con un $20 \%$ también en el sexo femenino lo que hace inferir que el sexo femenino se encuentra más vulnerable a iniciar las relaciones sexuales de forma precoz y en tercer lugar la falta o mala comunicación en los padres en el sexo masculino con un $16.66 \%$. Otras causas que favorecen el inicio precoz de las relaciones sexuales son los medios de comunicación con un $13.33 \%$ en el sexo masculino entre ellos el internet "redes sociales", la radio y televisión que cada día se encarga de enviar mensajes subliminales a los adolescentes, la presión social con 13.33\% en el sexo masculino que ejercen los grupos en las aulas de clases donde interactúan la mayoría del tiempo. 


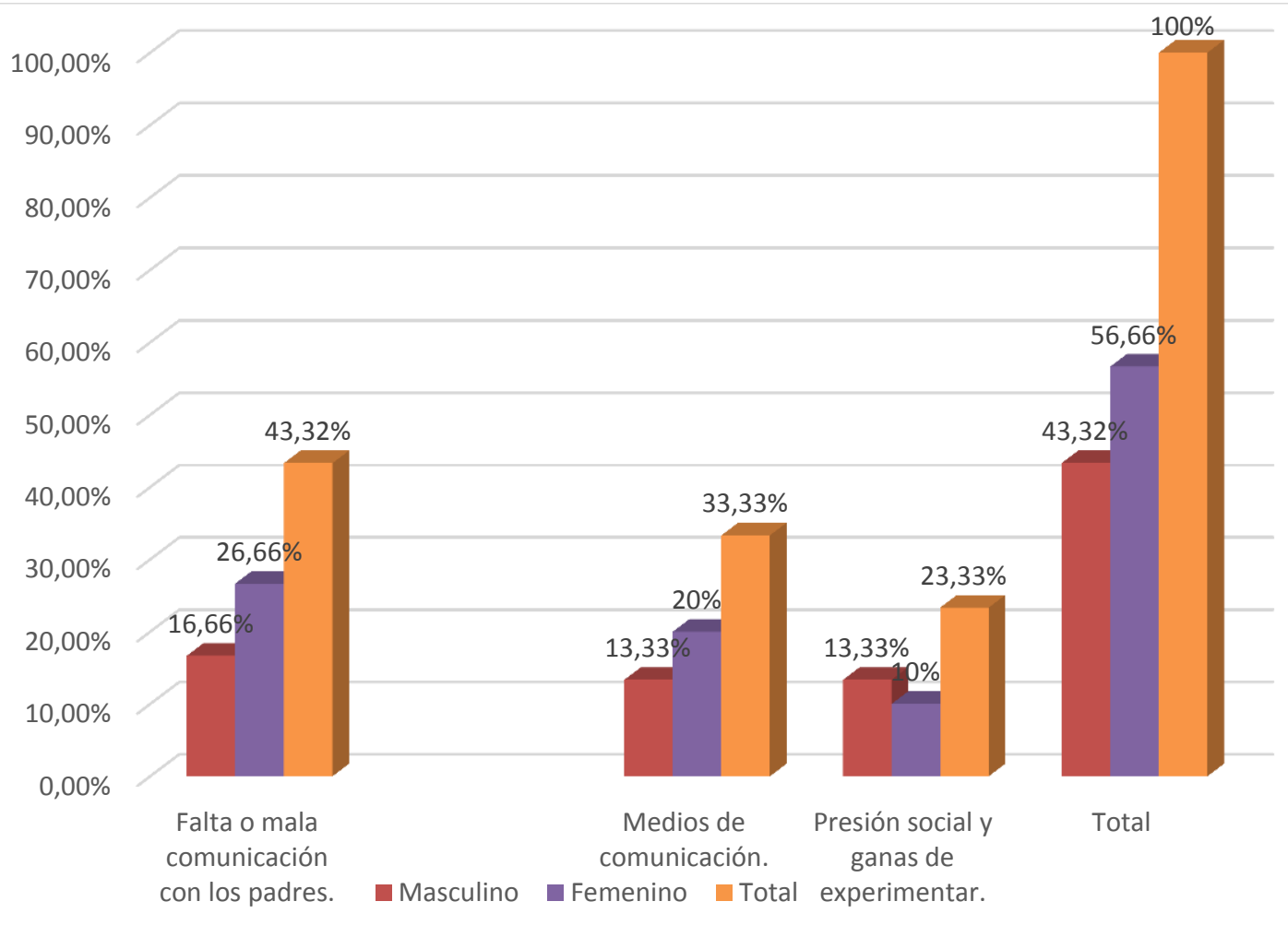

Gráfico 3. Factores que favorecen el inicio precoz de relaciones sexuales de los adolescentes de la comunidad el Carmen. 2018.

Los adolescentes no conocen las consecuencias del inicio temprano de las relaciones, solo el $46.67 \%$ afirmaron que el embarazo precoz es una de las consecuencias. El impacto del embarazo en la adolescencia es psicosocial y se traduce en deserción escolar, mayor número de hijos, desempleo, fracaso en la relación de pareja e ingresos inferiores de por vida. Contribuye a perpetuar el ciclo de la pobreza y la "feminización de la miseria". El embarazo en la adolescencia representa un serio problema social, y se considera desde el punto de vista médico y social como una situación de riesgo por ello la importancia de dar a conocer información oportuna y eficaz que demore el inicio de las relaciones sexuales en los adolescentes, otra de las consecuencias que conocen los adolescentes son las enfermedades de transmisión sexual con un $13.3 \%$ esto demuestra que a pesar de que el conocimiento de la existencia de métodos anticonceptivos es universal en mujeres entre los 13-29 años, sólo 15\% ha usado un método alguna vez en su vida y $10 \%$ lo usan en su primera relación sexual. (Gráfico 4). 


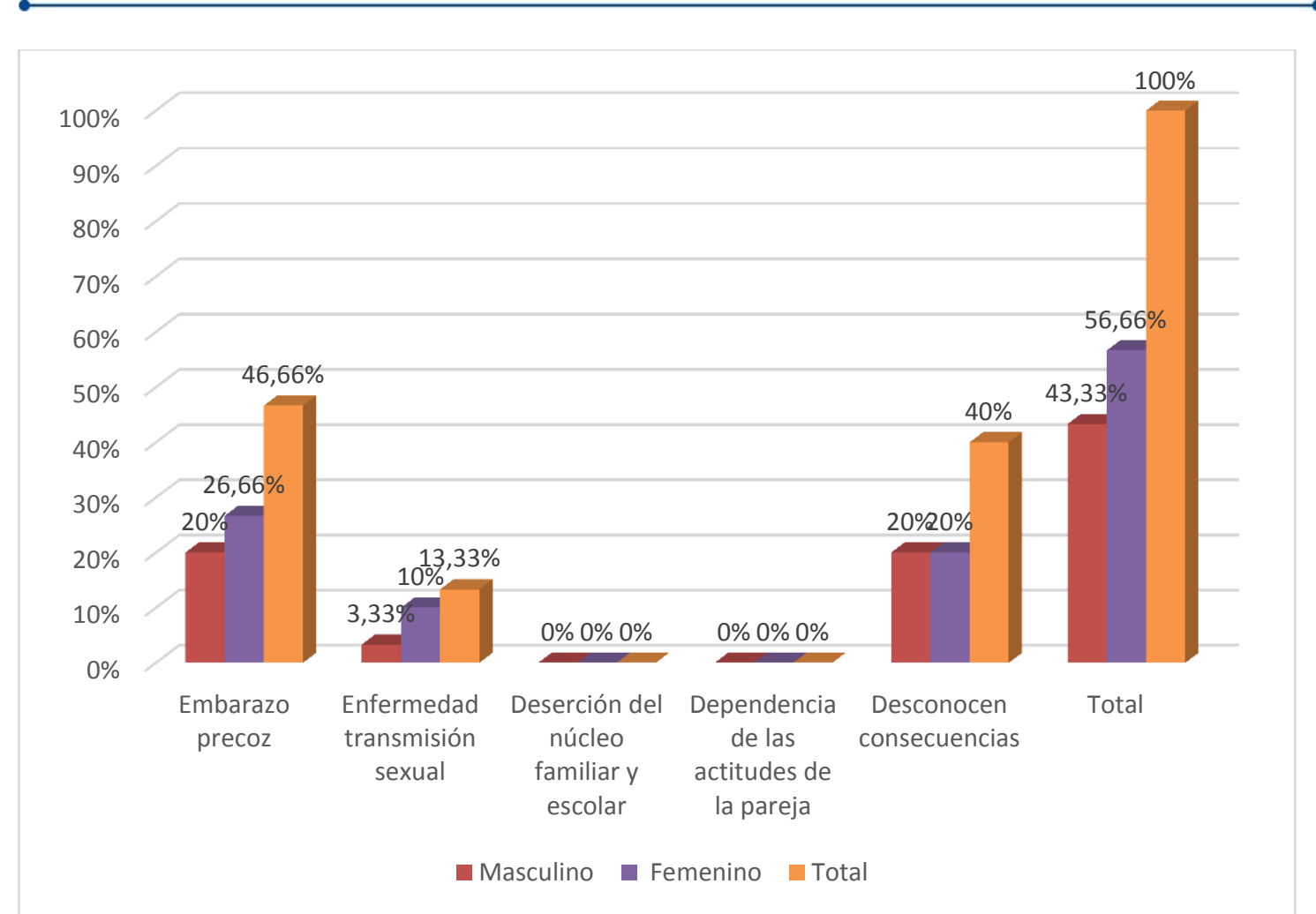

Gráfico 4. Conocimiento de las consecuencias del inicio precoz de las relaciones sexuales de los adolescentes con edades comprendidas entre 13 y 16 años de la comunidad el Carmen. 2018. Pretest.

Para cerrar, se observó que antes de la intervención el $33.33 \%$ de los adolescentes, tuvieron un conocimiento adecuado, seguido de un $66.66 \%$ de adolescentes que tuvieron un conocimiento inadecuado. Después de la intervención se logró elevar el porcentaje dando como resultado que el 89.99 de los adolescentes tuvieron conocimientos adecuados. Posterior a la intervención educativa según su edad, arrojaron que el $89,9 \%$ de ellos tuvieron un conocimiento adecuado y en $9.9 \%$ obtuvo un nivel de conocimiento inadecuado acerca de las consecuencias que acarrean el inicio precoz de las relaciones sexuales (Gráfico 5). Por tanto, se puede deducir que la intervención educativa realizada a los adolescentes tuvo el efecto de aumentar los conocimientos inadecuados con respecto a la salud sexual y principales consecuencias de iniciar de manera temprana sus relaciones sexuales. 


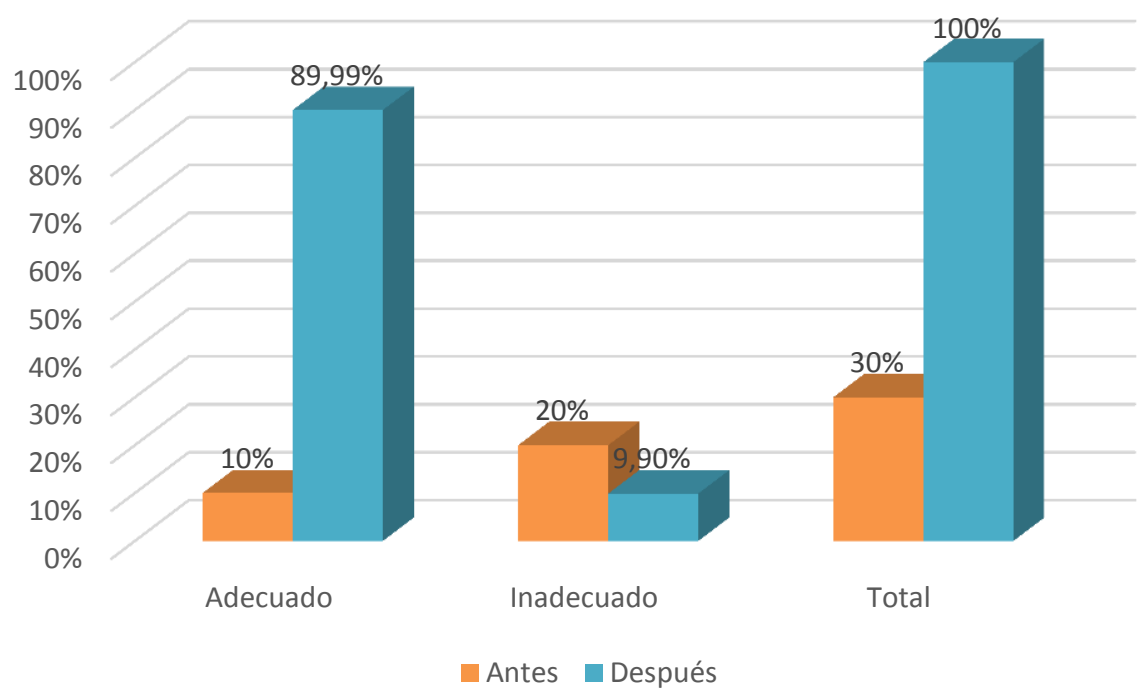

Gráfico 5. Nivel de conocimiento sobre las consecuencias del inicio precoz de las relaciones sexuales de los adolescentes con edades comprendidas entre 13 y 16 años de edad de la comunidad el Carmen. 2018. Pre y Post-test

\section{Discusión}

La adolescencia es una época en que los jóvenes asumen nuevas responsabilidades y experimentan cierta sensación de independencia, buscan su identidad, poner en práctica valores aprendidos en su infancia y a desarrollar habilidades que les permitirán convertirse en adultos. Al recibir el apoyo y el aliento de los adultos, los adolescentes se convierten en miembros plenos de sus familias y comunidades $\mathrm{y}$ dispuestos a contribuir (6).

La adolescencia es un periodo donde se toman muchas decisiones y se percibe la necesidad de obtener experiencias variadas, es esa búsqueda de identidad una de las razones que lo conlleva a un inicio temprano de las relaciones sexuales. Son muchos los factores que se han asociado con el inicio de la actividad sexual temprana, factores individuales como sexo masculino, propia toma de decisiones, papel que desempeña la madre en la educación sexual de sus hijos y la influencia de pares o amigos en la toma de decisiones, se asocian con el inicio temprano de la actividad sexual entre adolescentes (7).
La aprobación de las relaciones sexuales por parte de los adolescentes se ve influenciada por su ambiente y contexto social donde se desenvuelven. El inicio de la actividad sexual está determinado por un número reducido de factores que involucran la influencia del contexto social y la percepción que tienen los adolescentes de la presión existente por parte de sus padres y de sus amigos para tener o no relaciones sexuales a su edad (8).

Cuando los adolescentes inician su vida sexual activa sin contar con una educación sexual amplia, responsable y precisa, se pueden ver abocados a circunstancias que comprometan su salud física $y$ mental $y$ de esta manera comprometer su futuro sin el conocimiento adecuado de las consecuencias $(9,10)$. Una adecuada educación sexual en esta etapa del desarrollo resulta de vital importancia, debido a que el poco o inadecuado conocimiento genera conductas sexuales de riesgo que conllevan situaciones como los embarazos no deseados y el contagio de infecciones de transmisión sexual (ITS) (11). 
El inicio precoz de las relaciones sexuales no sólo es importante porque puede implicar falta de conocimientos adecuados al respecto, sino también porque el desarrollo psicológico y físico aún conlleva retos importantes para el individuo, funciones cognitivas implicadas en la toma decisiones, percepción de riesgo, planificación de actividades y control de impulsos aún están en proceso de maduración (12).

Durante los últimos 20 años los adolescentes han ganado el acceso a una amplia variedad de fuentes de información y desinformación sexual, de ahí la necesidad de mantener las acciones educativas dirigidas a incrementar sus conocimientos en la esfera de la sexualidad (13).

En vista de la necesidad de informar a la colectividad sobre las consecuencias de relación sexual precoz en adolescentes de la comunidad el Carmen Municipio Iribarren Parroquia Unión, se les ofreció herramientas como la observación, discusión grupales y talleres con la debida autorización de los padres o representantes, ya que los adolescentes, participaron de forma espontánea, orientada según su interés superior de aprendizaje respetando su libre consentimiento, como tal cual se expone en los Artículos: 43, 50, 67 de la LOPNNA, explicados, ya que "Todos los niños y adolescentes tienen derecho a ser informados y educados de acuerdo a su desarrollo, en salud sexual y reproductiva para una conducta sexual y una maternidad y paternidad responsable, sana voluntaria y sin riesgos" basada en una información oportuna y veraz (14).

Así mismo, de acuerdo a la LOPNA (Ley Orgánica de Protección Integral del Niño y Adolescente) todos los y las niños (as) y adolescentes tienen el derecho a ser informados, a participar activamente en su proceso educativo, brindando información y formación apropiada sobre la materia a los adolescentes. Al igual tienen derecho a recibir, buscar y utilizar todo tipo de información que sea acorde con su desarrollo y a seleccionar libremente el medio y la información a recibir (13).

Al considerar la información que poseían antes de los talleres y las respuestas obtenidas posteriores a conversaciones, talleres e intercambio de opiniones e información, se obtuvo que los adolescentes manejaban una información correcta en cuanto al inicio precoz de las relaciones sexuales y las consecuencias y riesgos que esto acarrea para sus vidas.

\section{CONCLUSIONES}

$\mathrm{L}$ a mayoría de los adolescentes objetos de la muestra iniciaron sus relaciones sexuales sin tener en cuenta las consecuencias que derivarían de sus actos sin la madurez emocional necesaria para manejar las consecuencias y prevenir los riesgos. En cuanto a los factores claramente relacionados con la precocidad sexual se detectó la falta de comunicación y una mala relación con sus padres, no obstante, el embarazo y las enfermedades de transmisión sexual son las situaciones de riesgo que asocian los adolescentes como consecuencia de iniciar una práctica temprana de relaciones sexuales, su edad de inicio es en su mayoría entre los 15 y 16 años de edad.

La intervención educativa fue favorable en tanto se evidenció en el pretest el mayor número de adolescentes tenían un conocimiento deficiente sobre las principales consecuencias de iniciar relaciones sexuales a temprana edad y luego al aplicar el post-test se detectó aumento del porcentaje de respuestas acertadas con lo cual puede haberse contribuido a una conducta responsable de los jóvenes en torno a la sexualidad. 
- Conflicto de intereses. Ninguno declarado por la autora

- Financiación. Ninguna declarada por la autora

- Investigación apegada a los códigos de ética establecidos con asentimiento $\mathrm{y}$ consentimiento informado de parte de los involucrados para la realización de la presente investigación.

\section{REFERENCIAS}

1.- Fondo de las Naciones Unidas para la Infancia (UNICEF). Adolescencia una etapa fundamental. UNICEF Nueva York. 2002. (Consultado el 3 enero de 2018). Disponible en: https://www.unicef.org/pub_adolescence_ sp.pdf

2.- Organización Mundial de la Salud WHO. Desarrollo de la adolescencia. (Consultado el 3 de enero de 2018). Disponible en: https://www.who.int/maternal_child_adol escent/topics/adolescence/dev/es/

3.- Mendoza L, Claros D, y Peñaranda C. Actividad sexual temprana y embarazo en la adolescencia: estado del arte. Rev. chil. obstet. ginecol.2016; 81(3):243-253

4.- Morales F, Camps E, Lorenzo U. Cuestionario de Madurez Psicológica. España; TEA Ediciones: 2012.

5.-Observatorio Venezolano de los Derechos Humanos de las Mujeres. Informe sobre la Salud Sexual en Venezuela. 2009. (Consultado el 20 de diciembre de 2017). Disponible en: http://observatorioddhhmujeres.org/mod ulos/informe $\% 20$ sobre $\% 20$ sit $\% 20$ ssr $\% 20$ en $\% 20$ venezuela $\% 202008 \% 204 \% 20$ abril $\% 201 . h t m$

6.- Pérez A. Guía de atención al adolescente. Sociedad Andaluza de Medicina Familiar y Comunitaria. 2015. (Consultado el 4 de enero de 2018). Disponible en: https://www.samfyc.es/pdf/GdTAdol/201 5009.pdf
7.-Holguín Y, Mendoza M, Esquivel C, Sánchez R, Daraviña A, y Acuña M. Factores asociados al inicio de la actividad sexual en adolescentes de Tuluá, Colombia. Rev. Chil. Obstet. Ginecol. 2013; 78(3):209-219

8.-Vargas E, Barrera F. ¿Es la autoestima una variable relevante para los programas de prevención del inicio temprano de la actividad sexual? Acta Colomb Psicol. 2005; 13:133-61

9.- Rodríguez A, Sanabria G, Contreras ME, Perdomo B. Estrategia educativa sobre promoción en salud sexual y reproductiva para adolescentes y jóvenes universitarios. Rev Cuba salud pública. 2013; 39(1):16174

10.- Barceló R, Navarro E. Conocimientos, actitudes y habilidades de los adolescentes escolarizados del sector público de barranquilla (Colombia) relacionados con su sexualidad. Salud Uninorte. 2013; 29(2):298-314

11.- Rodríguez Y, Oliva J, Gil A. La sexualidad en los adolescentes: algunas consideraciones. Rev AMC. 2007; 11(1):3445

12.- Cardona D, Ariza-Gerena A, GaonaRestrepo C, Medina-Pérez 0. Conocimientos sobre sexualidad en adolescentes escolares en la ciudad de Armenia, Colombia. Rev AMC 2015; 19(6):568-576

13.- Gómez R, Díaz M, Sainz E, Gómez K. Acciones educativas sobre aspectos de la sexualidad dirigidas a adolescentes. Rev Cub Salud Pública. 2015; 41(1). [Citado 2018 mayo 03]; Disponible en: http://scielo.sld.cu/scielo.php?script=sci_a rttext\&pid=S0864 34662015000100006\&lng=es.

14.- Asamblea Nacional. Venezuela. Ley Orgánica para la Protección del Niño y del Adolescente (LOPNA). Gaceta Oficial $\mathrm{N}^{\circ} 5$. 266 Extraordinario de fecha 2 de octubre del año 1998. (Consultada el 4 de enero de 2018). Disponible en: http://www.ucv.ve/uploads/media/lopna. pdf 